\title{
Conductors, semiconductors, and insulators irradiated with short-wavelength free-electron laser
}

\author{
J. Krzywinski, ${ }^{\text {a) }}$ R Sobierajski, and M. Jurek \\ Institute of Physics, Polish Academy of Sciences, Al. Lotników 32/46, PL-02-668 Warsaw, Poland \\ R. Nietubyc \\ Soltan Institute for Nuclear Studies, PL-05-400 Swierk, Poland \\ J. B. Pelka \\ Institute of Physics, Polish Academy of Sciences, Al. Lotników 32/46, PL-02-668 Warsaw, Poland \\ L. Juha, ${ }^{\text {b) }}$ M. Bittner, V. Létal, and V. Vorlíček \\ Institute of Physics, Czech Academy of Sciences, Na Slovance 2, 18221 Prague 8, Czech Republic
}

\begin{abstract}
A. Andrejczuk
Institute of Experimental Physics, University of Bialystok, Lipowa 41, PL-15-424 Bialystok, Poland

J. Feldhaus, B. Keitel, E. L. Saldin, E. A. Schneidmiller, R. Treusch, and M. V. Yurkov

Deutsches Elektronen-Synchrotron DESY, Notkestrasse 85, D-22603 Hamburg, Germany
\end{abstract}

(Received 11 August 2006; accepted 7 December 2006; published online 23 February 2007)

\begin{abstract}
The results of a study of irreversible changes induced at surfaces of metals, semiconductors, and insulators by extreme ultraviolet $(\lambda<100 \mathrm{~nm})$ ultrashort pulses provided by TESLA Test Facility Free-Electron Laser, Phase 1 (TTF1 FEL) are reported and discussed. The laser was tuned at 86, 89, and $98 \mathrm{~nm}$ during the experiments reported here. Energy spectra of ions ejected from the irradiated surfaces are also reported. Special attention is paid to the difference in the ablation behavior of (semi)conductors and insulators that we have observed. The difference is dramatic, while the absorption coefficients are similar for all materials at the TTF1 FEL wavelength. () 2007 American Institute of Physics. [DOI: 10.1063/1.2434989]
\end{abstract}

\section{INTRODUCTION}

Before the experiments on free-electron laser interaction with solids ${ }^{1}$ (FELIS) were performed at TESLA Test Facility Free-Electron Laser, Phase 1 (TTF1 FEL) in 2001 and 2002, the shortest wavelengths at which laser ablation had been investigated were 46.9 and $125 \mathrm{~nm}$. The $46.9 \mathrm{~nm}$ radiation was emitted from a capillary-discharge $\mathrm{Ne}$-like $\mathrm{Ar}$ extreme $\mathrm{UV}$ (XUV) laser, ${ }^{2}$ and the $125 \mathrm{~nm}$ radiation was generated by four-wave-sum-frequency mixing (FWSFM) of a $626 \mathrm{~nm}$ dye laser and its second harmonics in $\mathrm{Hg}$ vapor. ${ }^{3}$ The pulse duration in both cases was in the nanosecond range. A few papers (for a review see Refs. 4 and 5) have reported ablation induced at even shorter wavelengths (i.e., in the soft x-ray region), but the radiation used was not coherent. In the present study, not only the laser wavelength is short $(<100 \mathrm{~nm})$ but also the pulse duration is very short (fluctuates within $30-150 \mathrm{fs}$ ). ${ }^{6}$ The short-wavelength and ultrafast coherent beam represents a unique combination. The ablation behavior of a wide variety of materials has been investigated under these irradiation conditions.

In this article, key results of a study of irreversible changes induced on surfaces of metals, semiconductors, and insulators by ultrashort XUV pulses generated at the TTF1 FEL are reported and discussed. We also report the mass, energy, and spectra of ions ejected from the irradiated sur-

\footnotetext{
${ }^{a)}$ Electronic mail: jacek.krzywinski@desy.de

b) Author to whom correspondence should be addressed; electronic mail: juha@fzu.cz
}

faces. Special attention is paid to the difference in the ablation behavior observed for (semi)conductors and insulators.

\section{EXPERIMENT}

The samples were irradiated by the self-amplified spontaneous emission free-electron laser (SASE-FEL) beam emitted by the TTF at DESY, Hamburg. ${ }^{6}$ The wavelength was tuned between 86 and $98 \mathrm{~nm}$. Spectral and energy characteristics of the laser beam were measured using photon diagnostics described in detail elsewhere. ${ }^{7}$ The beam was focused into the interaction chamber by an elliptical mirror; for more details about the chamber and focusing, see Refs. 8 and 9 Morphological changes on the surfaces of exposed samples were investigated by Nomarski, conventional optical, scanning electron, and atomic force (AFM) microscopies. Raman spectra were taken with a laser microbeam in the usual backscattering geometry. This enables selected regions of the sample surface to be probed. Ion energies and mass spectra were measured using a time-of-flight (TOF) spectrometer equipped with high pass energy filter. A number of different samples were irradiated by the focused FEL beam. The bulk samples were $\mathrm{Au}, \mathrm{Si}$, poly(methyl methacrylate) (PMMA), Ce:YAG (YAG denotes yttrium aluminum garnet, $\mathrm{Y}_{3} \mathrm{Al}_{5} \mathrm{O}_{12}$ ), and $\mathrm{SiO}_{2}$ (quartz). We have also irradiated Si monocrystalline chips polished to optical quality and covered with thin films: $15 \mathrm{~nm} \mathrm{Au}(\mathrm{Au}-15)$ and 10 and $40 \mathrm{~nm}$ amorphous carbon $(a-\mathrm{C}-10$ and $a-\mathrm{C}-40)$. 


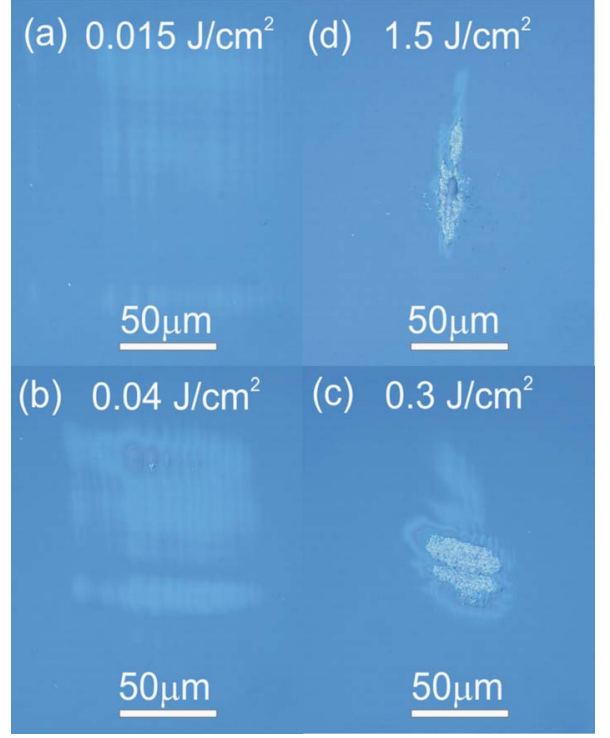

FIG. 1. (Color online) FEL-induced modification of monocrystalline silicon surfaces imaged by Nomarski microscopy.

\section{RESULTS AND DISCUSSION}

Modification of silicon surfaces irradiated with $89 \mathrm{~nm}$ FEL radiation of increasing fluence was recorded by a Nomarski microscope and is presented in Fig. 1. Each spot was irradiated with 11 shots. The first changes seen are due to the modification of the refractive index. An imprinted interference pattern, due to diagnostics installed in front of the focusing mirror and to slope errors of the mirror surface, is clearly seen. The AFM and TOF measurements do not indicate any morphological changes or ion emission, respectively. However, Raman spectra show signs of an amorphous phase in the modified region. The Raman spectra measured at three different locations are shown in Fig. 2. The amorphous fraction increases towards the center of the crater. The first observable changes were recorded at a fluence of $0.005 \mathrm{~J} / \mathrm{cm}^{2}$. In Table I, we present two different fluence thresholds at which we start to record modifications of the irradiated surface. The first $(\Phi \mathrm{I})$ is the fluence at which we notice a change of the refractive index, and the second (ФII) is the fluence at which deformation of the surface is indicated by the AFM. For metals and transparent materials, we have not found any difference between these two thresholds. It does not necessarily mean that the FEL radiation is not able to induce any irreversible change in the material, e.g., amor-

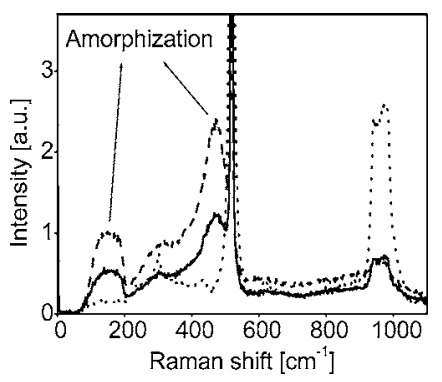

FIG. 2. Raman spectra measured at different places on a FEL-irradiated sample of monocrystalline Si (dotted line, unirradiated surface; solid line, crater rim; and dashed line, crater center).
TABLE I. Surface modification thresholds of various materials exposed to TTF1-FEL pulses.

\begin{tabular}{lcc}
\hline \hline \multicolumn{1}{r}{ Material } & $\Phi \mathrm{I}\left(\mathrm{J} / \mathrm{cm}^{2}\right)$ & $\Phi \mathrm{II}\left(\mathrm{J} / \mathrm{cm}^{2}\right)$ \\
\hline $\mathrm{Au}$ & 0.02 & 0.02 \\
$\mathrm{Au}-15$ & 0.01 & 0.01 \\
$a-\mathrm{C}-40$ & 0.01 & 0.03 \\
$\mathrm{Si}$ & 0.005 & 0.04 \\
$\mathrm{SiO}_{2}$ & 0.03 & 0.03 \\
$\mathrm{Ce}: \mathrm{YAG}$ & 0.02 & 0.02 \\
PMMA & 0.01 & 0.01 \\
\hline \hline
\end{tabular}

phization or recrystallization. However, for both groups of materials mentioned above, these changes do not lead to significant optical properties' modification. A clear difference between the two thresholds was found for amorphous carbon and monocrystalline silicon. It has been proven that in both cases a laser irradiation can induce phase transitions leading to a new phase with optical properties remarkably different from pristine material, i.e., graphitic carbon ${ }^{10}$ and amorphous silicon ${ }^{11}$ phases.

It can be seen that, except for Au-10 and PMMA, the $\Phi$ II values oscillate at about $0.03 \mathrm{~J} / \mathrm{cm}^{2}$. This is in a good agreement with the results of initial experiments conducted at the TTF1 FEL tuned to $98 \mathrm{~nm}$, i.e., $0.07 \mathrm{~J} / \mathrm{cm}^{2}$ for $a$-C-40. ${ }^{12}$ This is of the same order as the fluence $F_{c}$ at which the absorbed energy per atom is equal to the cohesive energy $E_{c}$,

$$
F_{c}=E_{c} n / \alpha,
$$

where $n$ is the atomic density and $\alpha$ the absorption coefficient. For typical values, $E_{c} \sim 3 \mathrm{eV} /$ atom, $n \sim 5$ $\times 10^{22}$ atom $/ \mathrm{cm}^{3}, \quad \alpha \sim 10^{6} \mathrm{~cm}^{-1}, \quad$ and $F_{c} \approx 0.025 \mathrm{~J} / \mathrm{cm}^{2}$, which correspond to the values shown in Table I.

Results of the AFM investigations carried out on the monocrystalline $\mathrm{Si}$ and $\mathrm{SiO}_{2}$ samples are presented in Figs. 3 and 4. The $\mathrm{Si}$ and $\mathrm{SiO}_{2}$ samples were irradiated by 11 and 22 shots, respectively, at an average fluence of $0.07 \mathrm{~J} / \mathrm{cm}^{2}$. One can notice a dramatic difference in the morphology of both craters.

The silicon crater is covered by columnar structures, the peaks of which are located above the sample's surface. This morphology is very typical for silicon surfaces ablated by multiple laser shots (for example, see Refs. 13-15 and references cited therein). The column formation may simply occur in supercooled liquid $\mathrm{Si}$ as the recrystallization front propagates towards the surface at different rates in different locations. ${ }^{13}$ However, more complex pictures of their origin were also developed. ${ }^{14,15}$
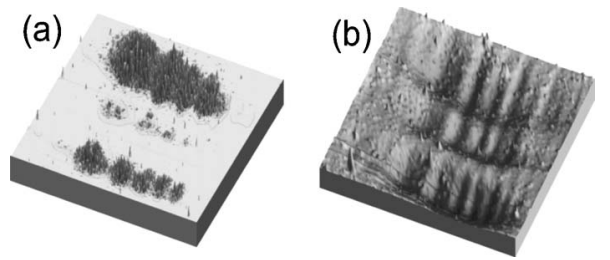

FIG. 3. AFM measurements of the silicon (a) and quartz (b) samples. Irradiation conditions were similar (fluence $\sim 0.07 \mathrm{~J} / \mathrm{cm}^{2}$ ). 

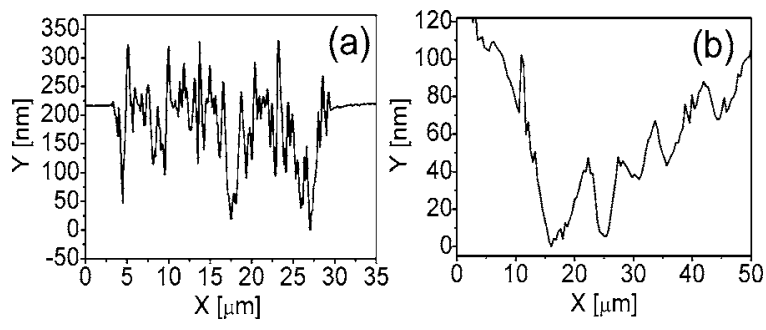

FIG. 4. Profiles of the craters shown in Fig. 3 for silicon (a) and quartz (b).

In the case of monocrystalline $\mathrm{SiO}_{2}$, the interior of the crater is very smooth and the interference pattern is clearly imprinted onto the sample's surface. Exposure of all investigated insulator samples to multiple shots leads to very similar surface patterns when the distance between the sample and the focal spot is the same. It has been demonstrated that the quality of ablated surfaces is very high, significantly better than surfaces ablated with ultrashort pulses at longer wavelengths, i.e., UV-Vis-NIR (where Vis and NIR denote visible and near IR, respectively) (see, for example, Refs. 16 and 17). A strong localization of the absorbed energy, i.e., both the attenuation and the thermal diffusion lengths in the chosen materials are exceptionally short for FEL pulses, is responsible for this difference. The direct, radiation-chemical action of $14 \mathrm{eV}$ photons on the structure of molecular solids could be partly responsible for this observation (i.e., only a portion of absorbed energy is thermalized). Here the direct action works together with the strong localization. For even shorter wavelengths, i.e., in the x-ray spectral region, and longer pulses, the quality of ablated surfaces often becomes poor due to the lack of near-surface localization of energy deposition. ${ }^{18-20}$ The morphology of the irradiated surface has a weak dependence on the FEL fluence. The dependence of ablation rate on fluence is shown in Fig. 5. For lower fluences the dependence follows the formula $d=l_{\mathrm{at}} \ln \left(\phi / \phi_{\mathrm{th}}\right)$, where $\phi$ is the laser fluence, $\phi_{\mathrm{th}}$ is the ablation threshold fluence, and $l_{\mathrm{at}}$ is the attenuation length of laser radiation in the material. Fitting the dependence gives a value of $l_{\text {at }}$ shorter than $10 \mathrm{~nm}$ that is in a good agreement with XUV optical constants of quartz reported in literature..$^{21}$ Both findings mentioned above indicate that intense ultrashort pulses of XUV radiation can be used for nano- and micromachining of insulators. The very small difference in ablation rate after 22 or 67 accumulated shots is shown in Fig. 5. Furthermore, there is surprisingly good agreement between the measured

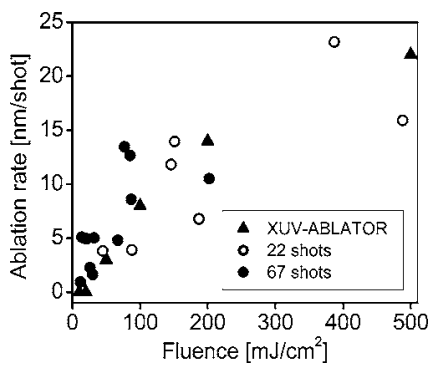

FIG. 5. Ablation rates in quartz irradiated by $86 \mathrm{~nm}$ FEL radiation as a function of laser fluence. Full circles, 67 shots accumulated; open circles, 22 shots accumulated; and triangles, the XUV-ABLATOR simulation.
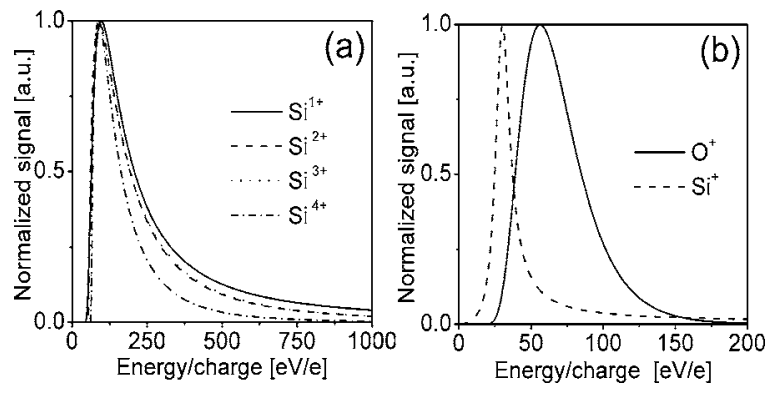

FIG. 6. The energy spectra of ions taken with the TOF spectrometer for the silicon (a) and quartz (b) samples. Signals have been normalized. The energy scale has been divided by the ions' charge state. Irradiation conditions were similar in both cases (fluence $\sim 1 \mathrm{~J} / \mathrm{cm}^{2}$ ).

data and the values calculated using the XUV-ABLATOR computer code (for more details about the code, Refs. 4 and 22-24), although the simulation can only be conducted for $100 \mathrm{ps}$ pulses and not the subpicosecond pulses used in the experiment. This agreement suggests that XUV radiationinduced ablation processes in insulators are dependent on fluence rather than intensity.

The ion spectra measured for the $\mathrm{Si}$ and $\mathrm{SiO}_{2}$ samples are presented in Fig. 6. Multiply charged ions were recorded from high-intensity irradiation of Si. The kinetic energy of the ions increased with charge state and reached the $\mathrm{keV}$ range for highly charged ions (up to 5+). Again, there is a clear difference between $\mathrm{Si}$ and $\mathrm{SiO}_{2}$. The high charge states and energetic ions shown for $\mathrm{Si}$ are typical of all (semi)conductors. However, only singly and doubly charged, lowenergy ions $(\sim 100 \mathrm{eV})$ were detected for the insulator $\mathrm{SiO}_{2}$ under all irradiation conditions. Other insulators investigated exhibited similar behavior. An interesting feature of the ion spectra observed for all (semi)conductors is that the measured values can be normalized to one curve if the energy of the ion is divided by its charge. This suggests a field emission mechanism.

The difference in ion energy spectrum of $\mathrm{Si}$ and $\mathrm{SiO}_{2}$ can be caused by two factors: the difference in the ionization potential and in the conductance. Because of the higher ionization potential of $\mathrm{SiO}_{2}$ with respect to $\mathrm{Si}$, the photoelectrons generated in $\mathrm{SiO}_{2}$ near the surface have low energy. This could limit their escape probability. Thus, a weaker field would be created at the $\mathrm{SiO}_{2}$, surface in comparison with $\mathrm{Si}$. The difference in the material conductances affects the plasma evolution at the surface. In the case of $\mathrm{SiO}_{2}$, there is no charge neutralization by the electrons coming from the interior of the sample. Coulomb forces prevent charged particles from escaping the thin layer of surface plasma. The numerical simulations of the charge distribution in the $\mathrm{SiO}_{2}$ sample have shown that the initial separation of the charges at the surface is in the order of a few angstroms. ${ }^{25}$ The ions and electrons cannot escape the evolving electric field until the plasma expands to the dimension comparable with the beam spot diameter. This state is reached in the nanosecond time scale when plasma approaches the thermal equilibrium. Hence one can expect a Maxwellian-type distribution of the measured ion energy spectrum in insulators. In the case of (semi)conductors, the return current can provide partial charge neutralization and the hot electrons are free to escape 
into the vacuum. Initially, ions remain relatively cold and stationary until the charge density decreases, and the Debye length becomes comparable to the plasma layer dimension. Evolving electric field can accelerate a fraction of the ions to high velocities. ${ }^{26,27}$ Since this effect is charge dependent, one can expect that ions with higher charges will be accelerated to higher velocities.

\section{CONCLUSIONS}

A distinct difference was observed between the behaviors of (semi)conducting materials and insulators irradiated by ultrashort XUV pulses generated at the TTF1 FEL. In the case of insulators, the morphology of the irradiated surface hardly depend on the beam intensity. In contrast, the irradiated silicon surface becomes very rough when the intensity exceeds the damage threshold. There is also a clear difference between insulators and (semi)conducting materials with respect to the spectra of ejected ions. Highly charged, energetic ions $(\sim$ a few $\mathrm{keV})$ were typical for (semi)conductors. Only singly and doubly charged, low-energy ions $(\sim 100 \mathrm{eV})$ were detected for insulators under all irradiation conditions.

\section{ACKNOWLEDGMENTS}

This work was funded by the Czech Ministry of Education within the framework of programs INGO (Grant No. 1P2004LA235) and National Research Centers (Projects LC510 and LC528), by the State Committee for Scientific Research of the Republic of Poland (Grant No. 72/E-67/SPB/ 5.PR UE/DZ 27/2003-2005), and by the European Commission (G1MA-CI-2002-4017; CEPHEUS).

${ }^{1}$ R. Sobierajski et al., Free Electron Lasers 2002, Argonne, edited by K.-J. Kim, S. V. Milton, and E. Gluskin (Elsevier, Amsterdam, 2003), p. II77.
${ }^{2}$ B. R. Benware, A. Ozols, J. J. Rocca, I. A. Artioukov, V. V. Kondratenko, and A. V. Vinogradov, Opt. Lett. 24, 1714 (1999).

${ }^{3}$ D. Riedel and M. C. Castex, Appl. Phys. A: Mater. Sci. Process. A69, 375 (1999).

${ }^{4}$ L. Juha et al., J. Microlithogr., Microfabr., Microsyst. 4, 0033007 (2005).

${ }^{5}$ L. Juha et al., Surf. Rev. Lett. 9, 347 (2002).

${ }^{6}$ V. Ayvazyan et al., Eur. Phys. J. D 20, 149 (2002).

${ }^{7}$ R. Treusch, C. Gerth, T. Lokajczyk, and J. Feldhaus, Nucl. Instrum. Methods Phys. Res. A 467-468, 30 (2001).

${ }^{8}$ A. Andrejczuk et al., HASYLAB Annual Report 2001, Pt. I, p. 117; http://hasylab.desy.de/science/annual_reports/hasylab_annual_reports/

index_eng.html

${ }^{9}$ R. Sobierajski et al., Rev. Sci. Instrum. 76, 013909 (2005).

${ }^{10}$ T. V. Kononenko, S. M. Pimenov, V. V. Kononenko, E. V. Zavedeev, V. I. Konov, G. Dumitru, and V. Romano, Appl. Phys. A: Mater. Sci. Process. A79, 543 (2004).

${ }^{11}$ Y. Izawa, Y. Setuhara, M. Hashida, M. Fujita, and Y. Izawa, Jpn. J. Appl. Phys., Part 1 45, 5791 (2006).

${ }^{12}$ B. Steeg, L. Juha, J. Feldhaus, S. Jacobi, R. Sobierajski, C. Michaelsen, A. Andrejczuk, and J. Krzywinski, Appl. Phys. Lett. 84, 657 (2004).

${ }^{13}$ T. H. Her, R. J. Finlay, C. Wu, S. Deliwala, and E. Mazur, Appl. Phys. Lett. 73, 1673 (1998).

${ }^{14}$ J. Bonse, S. Baudach, J. Kruger, W. Kautek, and M. Lenzner, Appl. Phys. A: Mater. Sci. Process. A74, 19 (2002).

${ }^{15}$ D. H. Lowndes, J. D. Fowlkes, and A. J. Pedraza, Appl. Surf. Sci. 154155, 647 (2000).

${ }^{16}$ S. Baudach, J. Bonse, J. Krüger, and W. Kautek, Appl. Surf. Sci. 154-155, 555 (2000).

${ }^{17}$ S. Küper and M. Stuke, Mater. Res. Soc. Symp. Proc. 129, 375 (1989).

${ }^{18}$ L. Juha et al., AIP Conf. Proc. 641, 504 (2002).

${ }^{19}$ L. Juha et al., Nucl. Instrum. Methods Phys. Res. A 507, 577 (2003).

${ }^{20}$ O. Maida, N. Kohma, M. Ueno, A. Shibuya, T. Kanashima, M. Okuyama, and H. Ohashi, Jpn. J. Appl. Phys., Part 1 40, 2435 (2001).

${ }^{21}$ http://www-cxro.lbl.gov/optical_constants/

${ }^{22}$ A. T. Anderson, Ph.D. thesis, University of California, Berkeley, 1996.

${ }^{23}$ A. T. Anderson, A. K. Burnham, M. T. Tobin, and P. F. Peterson, Fusion Technol. 30, 757 (1996).

${ }^{24}$ A. T. Anderson and P. F. Peterson, Exp. Heat Transfer 10, 51 (1997).

${ }^{25}$ R. Sobierajski, Ph.D. thesis, Warsaw University of Technology, 2005.

${ }^{26}$ F. Qian, V. Craciun, R. K. Singh, S. D. Dutta and P. P. Pronko, J. Appl. Phys. 86, 2281 (1999).

${ }^{27}$ M. Ye and C. P. Grigoropoulos, J. Appl. Phys. 89, 5183 (2001). 